
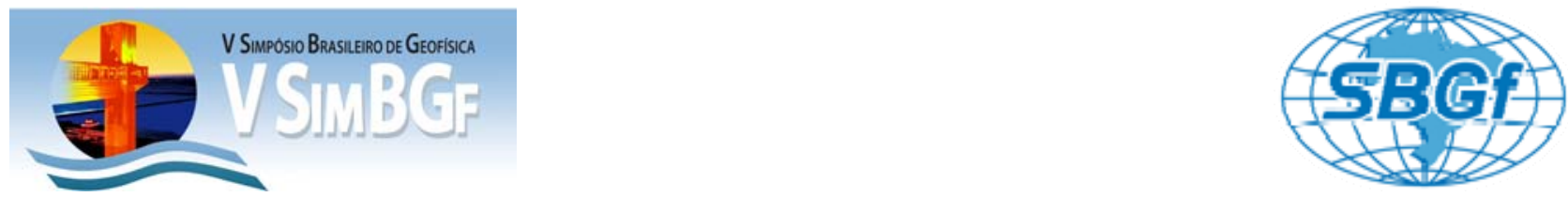

\title{
Parametrização de campo de velocidades sísmicas bidimensionais usando malha triangular
}

Luiz G. Guimarães ${ }^{\star^{1}}$, Hélcio M. Perin ${ }^{2}$, Wilson M. Figueiró ${ }^{3}$, Paulo E.M. Cunha ${ }^{4}$

LGG@peno.coppe.ufri.br

${ }^{1}$ COPPE-UFRJ, ${ }^{2}$ UNEB, ${ }^{3}$ CPGG-UFBA, ${ }^{4}$ CENPES-PETROBRAS

Copyright 2012, SBGf - Sociedade Brasileira de Geofísica

Este texto foi preparado para a apresentação no V Simpósio Brasileiro de Geofísica, Salvador, 27 a 29 de novembro de 2012. Seu conteúdo foi revisado pelo Comitê Técnico do V SimBGf, mas não necessariamente representa a opinião da SBGf ou de seus associados. É proibida a reprodução total ou parcial deste material para propósitos comerciais sem prévia autorização da SBGf.

\section{Abstract}

In this work we develop a simple methodology to the representation of irregularly distributed data (originally described by two dimensions) in order to map them in an unique dimension along an arc of curve (in particular as triangular functions periodically spaced). It means: it is identified, approximately, the points of a planar region to those belonging to a curve superimposed on the abovementioned region. We apply, with reasonable success, such method to the case of the two-dimensional compressional wave velocity field proceeding from the geologic model of the continental shelf break strongly marked by rugosities. Results show that not big scale variations can influence, in a sensible way, the quality of model representation.

Key-Words: Parameterization, Seismic Velocity Field, Geologic Model, Arc Length, and Triangular Mesh.

\section{Resumo}

Neste trabalho desenvolvemos uma metodologia simples para a representação de dados irregularmente distribuídos (originalmente descritos em duas dimensões) de tal modo que possam ser mapeados em uma única dimensão ao longo de um arco de curva (em particular como funções triangulares periodicamente espaçadas). Isto é, identifica-se, de modo aproximado, os pontos de uma região plana àqueles pertencentes a uma curva sobreposta à referida região. Aplicamos, com razoável sucesso, tal método ao caso do campo bidimensional de velocidades de ondas compressionais proveniente do modelo geológico da quebra da plataforma continental fortemente marcado por rugosidades. Resultados mostram que não grandes variações na escala podem influenciar, de modo sensível, a qualidade da representação do modelo.

Palavras-Chaves: Parametrização, Campo de Velocidades Sísmicas, Modelo Geológico, Comprimento de Arco e Malha Triangular.

\section{Introdução}

Estruturas geológicas reais são com freqüência representadas por modelos sintéticos (campos de velocidades sísmicas), tais como: Marmousi, SEG-EAGE e outros (Perin \& Figueiró, 2012 e Martinez \& Figueiró, 2011). Nestes casos, muitas vezes se faz relevante representar o campo de velocidades ao longo de todo o corpo geológico e isto é feito parametrizando o campo de velocidades em uma das mais diversas bases de funções tais como: matriciais, polinomiais, senoidais (Fourier), splines, ondaletas e outras (Bishop et al., 1985; Wyld, 1985; Figueiró, 2001; Dos Santos \& Figueiró, 2006; Dos Santos, 2008; Dos Santos \& Figueiró, 2011; e Santana, 2008). Entretanto, a obtenção de uma boa acurácia na aproximação pode exigir o uso de uma grande quantidade de parâmetros. Isto pode fazer com que o algoritmo de inversão se torne proibitivo devido alto custo computacional (Claerbout, 1992a; Claerbout, 1992b; Clapp \& Biondi, 1995; Etgen, 1997; Fomel, 1997; Nichols, 1994; e Schwab, 1997). Por exemplo, a Figura 1 mostra, em um mapa em escala de cinza, um modelo sintético para um campo de velocidades compressionais, $c(x, y)$, que correspondente a uma representação simplificada de um talude (quebra da plataforma continental) com extensão horizontal e profundidade normalizadas para 1 $\mathrm{km}$. Este simples modelo é gerado a partir de uma malha quadrada contendo aproximadamente $10^{4}=100 \times 100$ pontos. Nas representações convencionais de modelos $2 \mathrm{D}$, em geral, o número de parâmetros cresce com o quadrado de suas dimensões elevando o custo computacional, uma vez que a manipulação e armazenamento de dados cresce na mesma taxa. Neste trabalho pretendemos minimizar tal problema propondo uma metodologia para catalogar o campo de velocidade $c(x, y)$ ao longo de um arco de curva, como mostrará a seção seguinte.

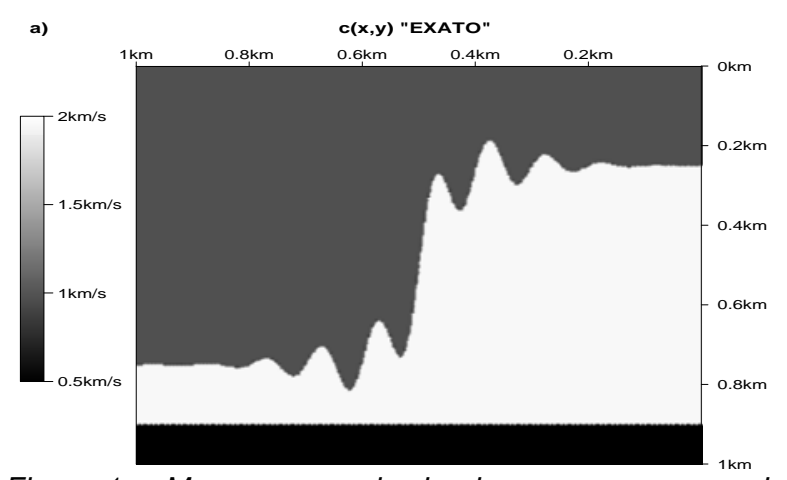

Figura 1 - Mapa em escala de cinza para o campo de velocidades $c(x, y)$.

\section{Mapeamentos bidimensionais ao longo de um arco}

As Figuras $2 a$ e $2 b$ representam funções triangulares periódicas usadas para um mapeamento $R \times R \mapsto R$ 
do tipo triangular de período $2^{-\mathrm{J}}$ (com $\mathrm{J}=3$ e $\mathrm{j}=5$, respectivamente), sendo $y_{J}$ descrita pela seguinte expansão em série de Fourier:

$y_{J}(x)=\frac{1}{2}-\frac{4}{\pi^{2}} \sum_{m=1}^{\infty} \frac{\cos \left[2^{(J+1)} \pi(2 m-1) x\right]}{(2 m-1)^{2}}$,

onde $m$ são os harmônicos da série de Fourier para esta função triangular periódica (Claerbout, 1997; Claerbout \& Fomel, 1997; Claerbout \& Nichols, 1994; Bednar, 1997; Claerbout, 1994; Fomel et al., 1997; Karrenbach, 1995; Schwab \& Claerbout, 1995; e Sod, 1985).

Outros tipos de funções podem ser usadas, com a mesma finalidade, para a geração de uma cobertura da região referente ao campo, por exemplo: senos ou cossenos, espirais, dente de serra, ou outras que não obedecem a uma forma matemática analítica definida. A escolha recaiu sobre a função triangular, pois esta não apresenta desvantagens tais como: distribuição irregular de pontos ao longo da curva com concentração deles nas proximidades dos picos da função (extremidades), que ficam perto das bordas no modelo, e escassez deles nas regiões mais internas do modelo, o que causa uma má amostragem de partes essenciais do campo (senos); incontinência da curva com respeito da região definida pelo modelo (espiral); presença de descontinuidades causadora de saltos que faz com que pontos distantes sejam colocados como vizinhos (dente de serra); dificuldade de obtenção de expressão matemática analítica (grande complexidade) ou inexistência de tal expressão para o comprimento de arco; e etc.

Segue da Figura 2c que podemos associar à malha, referente à função triangular, o comprimento de $\operatorname{arco} \Gamma_{\mathrm{J}}, \mathrm{a}$ saber:

$\Gamma_{J}(x)=x \sqrt{1+4^{(J+1)}}$.

Como o período de $y_{\mathrm{J}}$ é escalado segundo $2^{-\mathrm{J}}$, é interessante notar da Eq. (1) que a curva $y_{\jmath}$ oscila mais rapidamente a medida que $\mathrm{J}$ cresce. Isto é, a curva tornase cada vez mais densa sobre o campo, cobrindo-o de modo cada vez mais completo à medida que a freqüência de oscilação aumenta. Além disso, pode-se verificar pela Eq. (2) e pela Figura 2c um crescimento substancial do comprimento de arco de $\Gamma_{\mathrm{J}}$ com o aumento da escala $\mathrm{J}$. Como também mostram as Figuras $2 \mathrm{a}$ e $2 \mathrm{~b}$, este tipo de comportamento crescente, do comprimento de arco $\Gamma_{\mathrm{J}}$ com J, permite que a malha dada pela Eq. (1) faça um bom "recobrimento" do espaço $\mathrm{R}^{2}$ através do aumento da escala J. Assim, o campo de velocidade bidimensional é representado pelos pontos do arco unidimensional $\Gamma_{\mathrm{J}}$, isto é:

$$
F_{J}(x) \equiv c\left(x, y_{J}(x)\right) \text {. }
$$

Nas seções a seguir, vamos mostrar os resultados numéricos, discutir um pouco sobre vantagens e desvantagens dessa metodologia, bem como sumarizar nossas observações e discussões.

\section{MALHA TRIANGULAR: PERIODO $2^{`} \mathrm{~km}$}

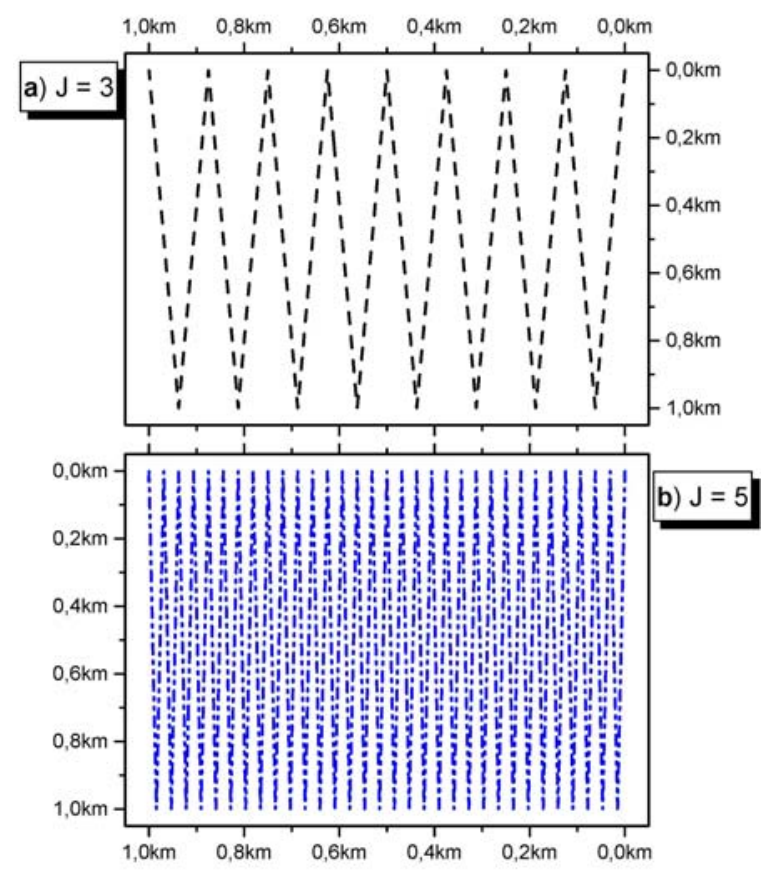

Figuras 2 - Exibição de $y_{\mathrm{J}}$ como uma malha do tipo função triangular de período $2^{-\mathrm{J}}, \mathrm{com} \mathrm{J}=3 \mathrm{em}$ (a) e $\mathrm{J}=5$ em (b).

\section{Resultados}

As Figuras 3a e 3b mostram o comportamento do campo unidimensional $F_{J}$ para as escalas $\mathrm{J}=3$ e $\mathrm{J}=5$, respectivamente. Cabe aqui salientar que neste tipo de mapeamento, o aumento da escala $\mathrm{J}$, afeta fortemente a magnitude dos gradientes do campo bidimensional original $c(x, y)$. Do ponto de vista numérico este é um efeito não desejado, pois pode introduzir singularidades espúrias que não existiam no campo original $c(x, y)$.

COMPRIMENTO DE ARCO DA MALHA DE PERIODO $2^{-1} \mathrm{~km}$

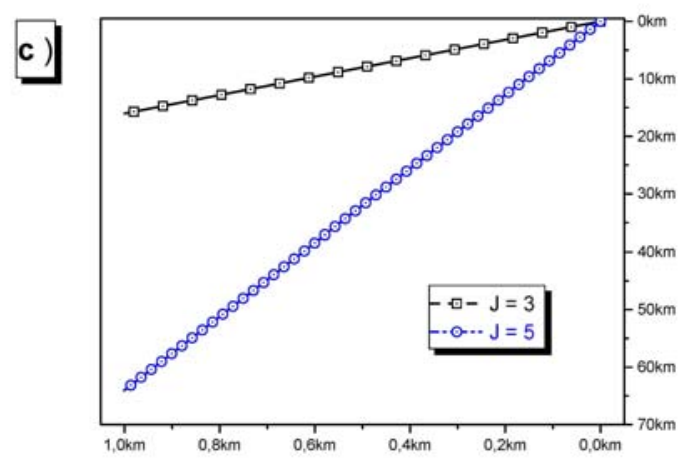

Figura 2c - Exibição, para duas diferentes escalas $\mathrm{J}$, do aumento do comprimento de arco de $\Gamma_{\mathrm{J}}$. 


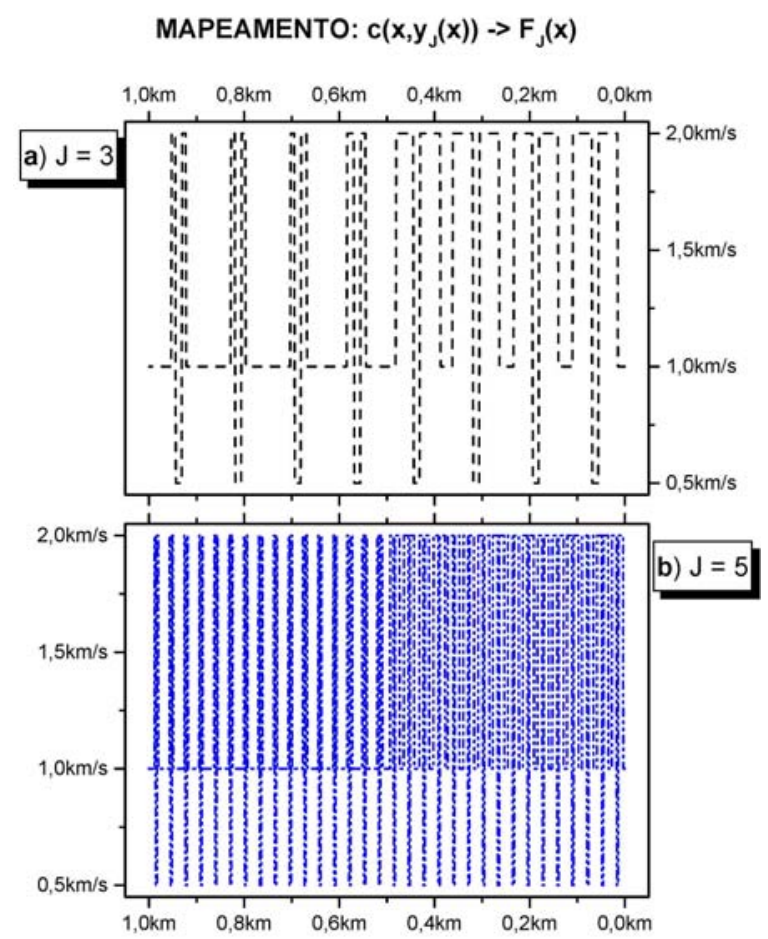

Figura 3 - Campo unidimensional de velocidades $F_{J}$ para as escalas $\mathrm{J}=3 \mathrm{em}$ (a) e $\mathrm{J}=5 \mathrm{em}$ (b).

Entretanto, ao fazermos o mapeamento inverso, as Figuras $4 a$ e $4 b$ sugerem que o efeito dominante é devido, principalmente, à escolha de uma escala adequada J para uma acurácia dada do problema. Estas Figuras mostram que na escala $\mathrm{J}=3$, o mapeamento ao longo de $\Gamma_{\mathrm{J}}$ apenas reproduz grosseiramente o perfil do campo original de velocidade. Entretanto, a Figura $4 \mathrm{~b}$ sugere que, na escala $J=6$, o perfil de velocidade $c(x, y)$ já está representado de forma bastante satisfatória.

\section{Discussões e Conclusões}

Visando representar um campo bidimensional de velocidades sísmicas de modo unidimensional sem significativa perda de acurácia, isto é: reduzir uma função de duas variáveis a uma outra de uma única variável (ou ainda: restringir uma função $2 \mathrm{D}$ a um subconjunto do domínio que possa ser descrito por uma única variável); mostramos neste resumo que é possível fazer, em caráter aproximativo e empírico, um mapeamento $R \times R \mapsto R$ de campos de velocidade bidimensionais ao longo de um arco com acurácia controlada. Para tanto, como mostram as Eqs. (1) e (3) é necessário parametrizar os valores do campo de velocidade ao longo de uma malha triangular (Figuras $3 a$ e 3b). Entretanto, apesar da acurácia da representação crescer com $2^{-\mathrm{J}}$, temos que nos precaver numericamente quanto à introdução de "singularidades espúrias", pois com o crescimento da escala $\mathrm{J}$ (fora de uma faixa de estabilidade), este tipo de mapeamento faz com que as funções amostradas tenham oscilações extremamente rápidas. Portanto, além da preocupação com a obtenção de uma escala J ótima, faz-se necessário, também, considerar a questão de sua estabilidade.

Até o momento a escolha das escalas está se dando de modo computacional e empírico, pois ainda não dispomos de um ferramental matemático capaz de realizar uma otimização analítica que permita associar a melhor representação possível do campo a um determinado valor ótimo da escala. Portanto, não há garantia de que a escala J, empiricamente obtida, tenha caráter local ou global. Deve-se acrescentar ainda que o aumento de $\mathrm{J}$ implica, num aumento do número de parâmetros do modelo, o que pode dificultar grandemente ou impossibilitar a resolução do problema inverso associado. O ideal que ainda continua sendo perseguido por este trabalho é aquele da obtenção do máximo de acurácia na representação (ou parametrização do modelo) utilizando-se o mínimo possível de parâmetros.

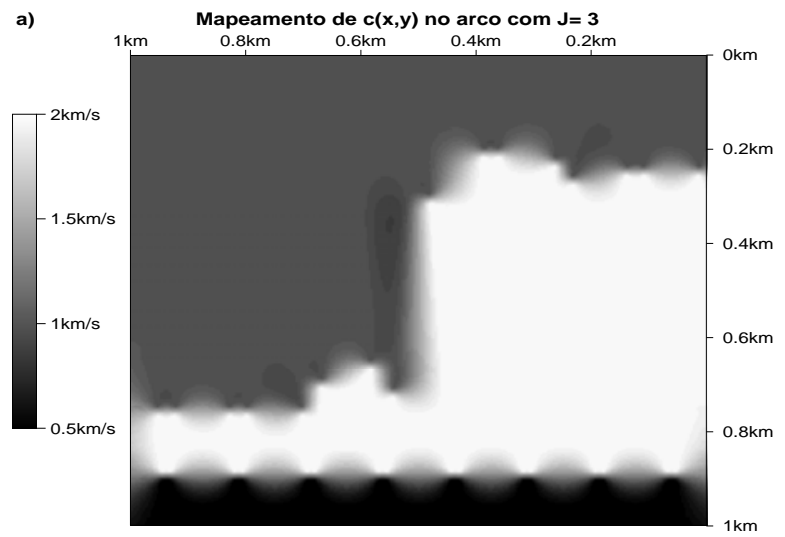

Figura 4a - Mapeamento inverso $F_{J} \mapsto C$ para a escala $\mathrm{J}=3$.

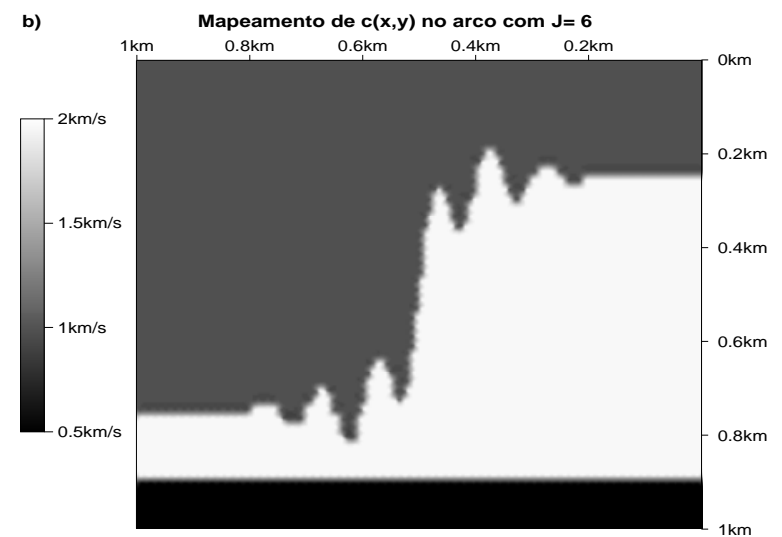

Figura $4 b$ - Mapeamento inverso $F_{J} \mapsto C$ para a escala $\mathrm{J}=6$. 


\section{Agradecimentos}

Os autores agradecem: à COPPE-UFRJ, à UNEB, ao CPGG-UFBA, à PETROBRAS e à ANP.

\section{Referências}

Bednar, J.B.; 1997. Least squares dip and coherency attributes. in SEP-95: Stanford Exploration Project, 219225.

Bishop, T.N.; Bube, K.P.; Cutler, R.T.; Langan, R.T.; Love, P.L.; Resnick, J.R.; Shuey, R.T.; Spindler, D.A. \& Wyld, H.W.; 1985. Tomographic determination of velocity and depth in laterally varying media. Geophysics, 50, 903-923.

Claerbout, J.F.; 1992a. Earth Soundings Analysis: Processing Versus Inversion. Blackwell Scientific Publications.

----; 1992b. Nonstationarity and conjugacy: Utilities for data patch work. in SEP-73: Stanford Exploration Project, 391-400.

----; 1994. Applications of Three-Dimensional Filtering. Stanford Exploration Project.

----; 1997. Multidimensional recursive filters via a helix. in SEP-95: Stanford Exploration Project, 1-13.

Claerbout, J.F. \& Fomel S.; 1997. Exploring threedimensional implicit wavefield extrapolation with the helix transform. SEP report, 95, 43-60.

Claerbout, J.F. \& Nichols, D.; 1994. Spectral preconditioning. in SEP-82: Stanford Exploration Project, 183-186.

Clapp, R.G. \& Biondi, B.; 1995. Multi-azimuth velocity estimation. in SEP-84: Stanford Exploration Project, 7588.

Dos Santos, M.A.B.; 2008. Modelagem Sismológica de Curvas Tempos de Trânsito com Parametrização por Série Trigonométrica do Campo Global de Velocidades. Trabalho de Graduação em Geofísica, UFBA, Salvador, BA.

Dos Santos, R.H.M. \& Figueiró, W.M.; 2006. Modelagem acústica bidimensional usando diferentes parametrizações de campos de velocidades. Revista Brasileira de Geofísica, ISSN 0102-261 X, vol. 24, no. 1, pags. 103-115.

Dos Santos, V.G.B. \& Figueiró, W.M.; 2011. Seismic ray tomography using $L_{1}$ integral norm. Revista Brasileira de Geofísica, ISSN 0102-261 X, vol. 29, no. 2, pags. 347358.

Etgen, J.; 1997. Problems and prospects in interval velocity estimation. Presented on Stanford Exploration Project Annual Meeting.
Figueiró, W.M., 2001. An analytical expression for degree of determination of geophysical model parameters. Boletim SBMAC, vol. 5.

Fomel, S.; 1997. On model-space and data-space regularization: A tutorial. in SEP-94: Stanford Exploration Project, 141-164.

Fomel, S.; Clapp, R. \& Claerbout J.; 1997. Missing data interpolation by recursive filter preconditioning. in SEP-95: Stanford Exploration Project, 15-25.

Karrenbach, M.; 1995. Elastic tensor wave fields. PhD thesis, Stanford University.

Martinez, A.C. \& Figueiró, W.M.; 2011. Parametrização ondaleta do campo de velocidades sísmicas relativo a um domo salino. Rio 2011 Expanded Abstracts, CD-ROM, 12th International Congress of the Brazilian Geophysical Society, CD-ROM, Rio de Janeiro, Brasil.

Nichols, D.; 1994. Velocity-stack inversion using Lp norms. in SEP-82: Stanford Exploration Project, 1-16.

Perin, H.M. \& Figueiró, W.M.; 2012. Parameterization of two-dimensional seismic velocity fields using Haar wavelet. 82th Annual Meeting of the Society of Exploration Geophysicists, Las Vegas, 2012 SEG Techical Program Expanded Abstracts.

Santana, J.L.S.; 2008. Diferentes Parametrizações do Campo de Velocidades Sísmicas do Modelo da Quebra da Plataforma Continental. Trabalho de Graduação em Geofísica, UFBA, Salvador, BA.

Schwab, M.; 1997. Cross product operator detects plane reflectors. in SEP-94: Stanford Exploration Project, 181188.

Schwab, M. \& Claerbout, J.; 1995. The interpolation of a 3-D data set by a pair of 2-D filters. in SEP-84: Stanford Exploration Project, 271-278.

Sod, G.A.; 1985. Numerical methods in fluid mechanics. Cambridge University Press. 